Psychotherapeut 2022 $\cdot 67: 34-41$

https://doi.org/10.1007/s00278-021-00565-8

Angenommen: 24. November 2021

Online publiziert: 18. Januar 2022

○ Der/die Autor(en) 2022

\section{Q Q}

\title{
Unterstützung für Kinder und Jugendliche mit psychisch erkrankten Eltern im „Children of mentally ill parents -network"
}

\author{
Bundesweites Forschungs-, Versorgungs- und \\ Implementierungsmodell
}

\author{
Silke Wiegand-Grefe \\ Klinik für Kinder- und Jugendpsychiatrie,-psychotherapie und -psychosomatik, Zentrum für Psychosoziale \\ Medizin, Universitätsklinikum Hamburg Eppendorf (UKE), Hamburg, Deutschland
}

\section{Zusammenfassung}

Kinder psychisch kranker Eltern sind als Risikogruppe für die Entwicklung eigener Auffälligkeiten bekannt. Gleichwohl weisen viele Unterstützungsangebote einen befristeten Projektcharakter auf, und es ist noch keine familienorientierte Regelversorgung für Kinder und Jugendliche mit psychisch erkrankten Eltern in Deutschland etabliert. Am Beispiel des Verbunds "Children of mentally ill parents network" (CHIMPS-NET) wird dargestellt, wie sich neue Versorgungsformen mit den Möglichkeiten des Innovationsfonds des Gemeinsamen Bundesausschusses (GB-A) in die Versorgung implementieren lassen. Das systematische Implementierungsmodell des Verbunds, basierend auf Arbeiten aus Australien und Norwegen, wird vorgestellt. Nach der Implementierung und bei erfolgreicher Evaluation ist eine Übernahme der bedarfsorientierten, familienorientierten Versorgungsformen des Verbunds in die regelhafte Versorgung geplant.

\section{Schlüsselwörter}

Familienorientierte Regelversorgung · Prävention · Psychotherapeutische Interventionen .

Implementierungsforschung · Evaluation

Etwa $15 \%$ der Bevölkerung leiden an psychischen Erkrankungen. Für die Betroffenen hält das deutsche Gesundheitssystem eine Vielzahl von Behandlungsangeboten in unterschiedlicher Intensität bereit - vom vollstationären Behandlungsangebot über teilstationäre Versorgungsmöglichkeiten bis hin zu ambulanten Therapieangeboten. Bislang wird bei der Behandlung jedoch wenig berücksichtigt, dass die gesamte Familie von einer psychischen Erkrankung betroffen sein kann. Das im Innovationsfond des Gemeinsamen Bundesausschusses (GB-A) geförderte "Children of mentally ill parents-network" (CHIMPSNET) verfolgt das Ziel, die bisherige Regelversorgung um familienorientierte neue Versorgungsformen zu erweitern.

\section{Hintergrund}

Psychiatrische Regelversorgung von Erwachsenen

Im stationären Behandlungskontext einer psychiatrischen Klinik für Erwachsene bleibt oft unberücksichtigt, dass die Patienten und Patientinnen auch Eltern sein können und minderjährige Kinder zu versorgen haben. Nicht regelhaft wird dies in der Familienanamnese erfragt. Im Verlauf der Behandlung in einer erwachsenenpsychiatrischen Klinikfinden nur vereinzelt Familiengespräche mit den Kindern statt. Häufig sind Stationen auch nicht darauf eingestellt, dass Kinder ihre Eltern besuchen. Es werden kaum Familienzimmer mit Spielzeugen oder familienfreundliche 
Wartebereiche bereitgehalten, in denen sich Kinder willkommen fühlen können. In der Mehrzahl der Fälle herrscht in erwachsenenpsychiatrischen Kliniken keine Willkommenskultur für minderjährige Kinder ihrer Patienten und Patientinnen. Mit Angehörigen sind im erwachsenenpsychiatrischen Kontext die Partner/-innen von Patientinnen und Patienten gemeint.

\section{Kinder und Jugendliche psychisch erkrankter Eltern}

\section{Epidemiologische Daten zu Entwicklungsrisiken und psychischen Auffälligkeiten}

Epidemiologische Schätzungen ergeben (z.B. Grube und Dorn 2007), dass etwa 10-30\% der psychisch erkrankten Mütter minderjährige Kinder haben. In Deutschland haben etwa 3 Mio. Kinder unter 18 Jahren einen psychisch erkrankten Elternteil, mit dem sie in der Mehrzahl der Fälle zusammenleben. Empirische Untersuchungen belegen das Entwicklungsrisiko dieser Kinder und Jugendlichen: Circa $60 \%$ der betroffenen Kinder entwickeln im Laufe ihres Lebens eine eigene psychische Erkrankung (Mattejat und Remschmidt 2008). Kinder psychisch erkrankter Eltern weisen 3-7-fach höhere Auffälligkeitsraten gegenüber Kindern gesunder Eltern auf (Wiegand-Grefe et al. 2009) und haben häufig eine schlechtere Lebensqualität (Radicke et al. 2021). Auch die Familienfunktionalität in Familien mit psychisch erkrankten Eltern kann beeinträchtigt sein; es lassen sich enge Zusammenhänge zum Befinden der Kinder psychisch erkrankter Eltern feststellen (Wiegand-Grefe et al. 2019; Sell et al. 2021). In der für Deutschland repräsentativen epidemiologischen BELLA-Studie ${ }^{1}$ ergeben sich für $21,9 \%$ aller Kinder und Jugendlichen Hinweise auf psychische Auffälligkeiten (Ravens-Sieberer et al.

\footnotetext{
1 BELLA steht für die „BEfragung zum seeLischen WohLbefinden und VerhAlten" und untersucht die psychische Gesundheit und gesundheitsbezogene Lebensqualität von Kindern und Jugendlichen in Deutschland. Die BELLA-Studie ist ein Zusatzmodul der Studie zur Gesundheit von Kindern und Jugendlichen in Deutschland (KIGGS), welche im Rahmen der Bundesgesundheitsberichterstattung am Robert-Koch-Institut durchgeführt wird.
}

2007). Steffen et al. (2018) fanden in einer Analyse von Krankenkassendaten, dass der Anteil der Kinder und Jugendlichen, die auf Jahresebene mindestens eine Diagnose einer psychischen Störung erhielten, von $23 \%$ im Jahr 2009 auf $28 \%$ im Jahr 2017 gestiegen war.

Die elterliche psychische Erkrankung kann sich in vielfältiger Weise auf die Entwicklung der Kinder auswirken. So kann die Eltern-Kind-Interaktion durch die Erkrankung beeinträchtigt sein. Etliche Studien zeigen Zusammenhänge insbesondere der frühen Eltern-KindInteraktionen mit der Entwicklung der Kinder auf. Beispielweise wurde in Untersuchungen der frühen Mutter-KindInteraktion deutlich, dass sich depressive Mütter ihren Säuglingen gegenüber passiver und weniger sensitiv verhalten als gesunde Mütter (Tronick und Reck 2009; Hatzinikolaou und Murray 2010). Externalisierende Verhaltensstörungen von Kindern und Jugendlichen sind wiederum mit einem Mangel an positivem Elternverhalten, wie Feinfühligkeit, emotionaler Zuwendung und Akzeptanz, verknüpft (Mäntymaa et al. 2004; Petermann und Petermann 2006; Smeekens et al. 2007). Kinder, deren Eltern eine Persönlichkeitsstörung aufweisen, zeigen die höchsten Auffälligkeitsraten und den ungünstigsten Entwicklungsverlauf (Wiegand-Grefe et al. 2011b). Über Interaktionsstörungen und eingeschränkte elterliche Erziehungskompetenzen hinaus sind die Kinder und Jugendlichen oft zusätzlichen psychosozialen Belastungsfaktoren ausgesetzt. So können chronische psychische Erkrankungen mit wiederholten Trennungserlebnissen oder schwierigen sozialen Lebenssituationen einhergehen. Bedeutsam ist auch das erhöhte Erkrankungsrisiko durch eine genetische Disposition, wie es z. B. bei Schizophrenie oder bipolaren Störungen von besonderer Relevanz ist.

\section{Versorgungssituation in Deutschland}

Trotz des erhöhten Erkrankungsrisikos und der vielfältigen Entwicklungsrisiken gibtes bislang noch keine regelhafte Versorgung für Kinder und Jugendliche psychisch erkrankter Eltern in Deutschland. Die meisten Angebote haben befristeten Projektcharakter und sind bislang zumeist nicht regelhaft etabliert oder richten sich nur an eine spezielle Zielgruppe, wie z.B. die Kinder depressiver Patienten und Patientinnen. Wieder andere Angebote richten sich explizit nicht an Kinder und setzen für die Teilnahmean Angehörigengruppen ein Mindestalter von 14 oder 16 Jahren voraus. Die Arbeit in einer der in Deutschland an einigen Stellen eingeführten Eltern-KindTageskliniken schildert beispielhaft die Arbeit von Müller et al. (2015). Für psychisch schwer kranke bzw. schwerstkranke Mütter, die einer vollstationären Behandlung mit spezifischer Fachkompetenz bedürfen, offenbarte eine Erhebung von Jordan et al. (2012) eine gravierende Unterversorgung um den Faktor 10. Dabei machen die Praxis und die Forschung deutlich, dass Kinder die Erkrankung ihrer Eltern wahrnehmen und für sich nach einem Erklärungsmodell suchen, was bisweilen sehr angstauslösend sein kann, wie die folgenden Fallbeispiele bestätigen (ausführlicher: Wiegand-Grefe und Taczkowski 2021).

Fallbeispiel 1. Der 11-jährige Tom erlebt seit Jahren die Krankheitsschübe seiner Mutter mit, die an einer paranoiden Schizophrenie leidet. Während des letzten Krankheitsschubs las der Junge heimlich die Packungsbeilagen der Psychopharmaka seiner Mutter, um sich Informationen zu beschaffen. Nach Lektüre der Nebenwirkungen vermutete er, dass seine Mutter an einer Herzerkrankung leide.

Fallbeispiel 2. Die 5-jährige Emma kennt seit Langem die Antriebslosigkeit ihres depressiven Vaters. Auf dem Weg zum ersten gemeinsamen Gespräch nach dem CHIMPS-Ansatz fragt sie ihre Mutter, ob der Papa Krebs habe, wie die Oma. Die habe auch immer im Bett gelegen und sei dann gestorben.

\section{Paradigmenwechsel - von der individuumszentrierten hin zur familienorientierten Versorgung}

Deutschland hat ein sehr weit entwickeltes, hoch spezialisiertes Versorgungssystem - aber: Es krankt daran, dass im Fokus aller Versorgungssysteme, sei es im Medizinsystem in der Behandlung von Erwachsenen oder von Kindern (geregelt im SGB V) oder in den Unterstützungs- 
maßnahmen im Jugendhilfesystem (geregelt im SGB VIII) individuumszentriert gearbeitet wird. Das sehr gute, jedoch individuumszentrierte Gesundheitssystem umfasst kaum familienorientierte Versorgung, z.B. Angebote zu Prävention und Therapie von Angehörigen chronisch und/ oder schwer erkrankter Menschen. Im Auftrag des jeweiligen Behandlers oder Unterstützers, der den Fokus und den Schwerpunkt der jeweiligen Interventionen definiert, steht immer der einzelne Mensch, sei es ein Erwachsender oder ein Kind (ausführlich: Wiegand-Grefe und Taczkowski 2021). Wenn ein Mensch schwer erkrankt ist, betrifft dies aber die ganze Familie. Alle Angehörigen, einschließlich der Kinder und Geschwister, sind durch eine schwere Erkrankung im Familiensystem hoch belastet, sei es eine schwere psychische oder eine körperliche Erkrankung eines Familienmitglieds. Und zwar gilt dies unabhängig davon, ob der erkrankte Mensch ein Elternteil oder ein Kind ist. Die Familie kommt mit ihren Sorgen und Fragen im Versorgungssystem aber kaum vor. Im Versorgungskontext werden die psychisch erkrankten Erwachsenen, auch die erkrankten Kinder betrachtet. Aber bislang werden die psychisch erkrankten Erwachsenen wenig in ihrer Rolle als Eltern wahrgenommen, als Mütter und Väter, die für ihre Kinder in vielerlei Hinsicht da sein müssen. Und die Kinder und Jugendlichen psychisch erkrankter Eltern werden bislang trotz erhöhtem eigenen Erkrankungsrisiko im Versorgungssystem bislang nicht ausreichend in der Regelversorgung berücksichtigt (Wiegand-Grefe und Taczkowski 2021). Familienorientierte Therapien sind im Versorgungssystem nicht vorgesehen es gibt keine Ressourcen der Behandler, keine Abrechnungsziffern, keine familienfreundlichen Wartebereiche etc. Familientherapie ist keine Krankenkassenleistung. Allenfalls werden Angehörige gelegentlich im Versorgungssystem berücksichtigt - so können einzelne Sitzungen mit Angehörigen als Angehörigengespräche, beispielsweise Sitzungen mit den Eltern im Rahmen der Psychotherapie eines Kindes, abgerechnet werden. Aber fachlich ist zwischen Angehörigenarbeit und konsequenter Familientherapie zu unterscheiden. Die Angehörigenarbeit dient der Unterstützung der Behandlung des Einzelnen, während
Familientherapie die ganze Familie im Blick hat. Die Loyalitäten und Identifizierungen der Behandler und Behandlerinnen mit ihrem Gegenüber sind jeweils sehr unterschiedlich. Diese eingeschränkte Perspektive aller Versorgungssysteme hat fatale Folgen und erhebliche, auch gesundheitsökonomische Konsequenzen. So könnte u. a. diese fehlende Familienorientierung wesentlich dazu beitragen, dass sich psychische Erkrankungen über die Generationen hinweg fortsetzen und von einer transgenerationalen Weitergabe ausgegangen werden muss (Wiegand-Grefe und Taczkowski 2021).

Es ist wünschenswert, dass sich die Familienorientierung stärker im Versorgungssystem etabliert. Einerseits könnte sich durch die Anerkennung der systemischen Therapie, die die Familie in ihrer Konzeptbildung der Systeme eher berücksichtigt, die Versorgung auch von Familien verändern und eine familienorientiertere Perspektive ins individuumszentrierte Versorgungssystem eingebracht werden. Andererseits gibt es derzeit durch den GB-A eine Förderpolitik, die möglicherweise die Versorgungslandschaft ebenfalls beeinflussen und hoffentlich diesbezüglich verbessern wird: den Innovationsfond. Im Innovationsfond des GBA werden seit 2016 Versorgungsansätze gefördert (sog. neue Versorgungsformen), die Versorgungslücken identifizieren und für diese Lücken eine Versorgung anbieten sollen (Wiegand-Grefe und Taczkowski 2021). Dies geschieht in Form von Einzelprojekten, regionalen Modellvorhaben oder auch großen bundesweiten Verbünden, wie sie die Verbünde für Familien mit Kindern mit einer seltenen Erkrankung (CARE-FAM-NET) oder für Kinder und Jugendliche mit psychisch kranken Eltern darstellen (CHIMPS-NET).

Der Verbund CHIMPS-NET wird beispielhaft für eine familienorientierte Versorgung von Kindern und Jugendlichen mit psychisch erkrankten Eltern in diesem Beitrag vorgestellt.

\section{Children of mentally ill parents - network}

Der im Innovationsfonds des GB-A finanzierte Verbund CHIMPS-NET verfolgt das Ziel, neue Versorgungsformen der gesetzlichen Krankenversicherung bereitzustellen, die die bisherige Regelversorgung erweitern sollen. Ausführlichere Informationen finden sich im Studienprotokoll (Wiegand-Grefe et al. subm.). Bei erfolgreicher Evaluation der 4 neuen Versorgungsformen des CHIMPS-NETVerbunds, CHIMPS-T, CHIMPS-MFT und CHIMPS-P sowie iCHIMPS, können diese in die regelhafte Versorgung überführt werden. Dabei steht CHIMPS-T für die therapeutische Intervention (Therapie, "therapy"), CHIMPS-MFT für die „Multifamilientherapie“ ("multifamily therapy“) und CHIMPS-P für eine kurze "Prävention" („prevention“) und iCHIMPS für die OnlineIntervention mit Jugendlichen. Die Interventionen und das methodische Design der randomisierten kontrollierten Evaluationsstudien sind in Studienprotokollen beschrieben (Dülsen et al. subm.; Wiegand-Grefe et al. subm.). Das heißt, über die Fördermittel des Innovationsfonds lassen sich die bestehenden Versorgungsstrukturen (hoffentlich) erweitern.

\section{Implementierungsperspektive}

Der Verbund CHIMPS-NET hat auch die Perspektive der Implementierung im Blick. Es wird heutzutage davon ausgegangen, dass nicht nur evidenzbasierte, in klinischen randomisiert-kontrollierten Studien („randomized controlled trials", RCT) evaluierte Interventionen für eine jeweils klar definierte Zielgruppe, hier die Kinder und Jugendlichen psychisch erkrankter Eltern, bereitstehen müssen. Auch die Prozesse der Implementierung selbst sollten im Fokus sein (Baumeister 2014; Petermann 2014). Hindernisse und Barrieren der Implementierung sollten verstanden und geebnet, Zugangswege zur Versorgung gebahnt werden. Mittlerweile hat sich auch in Deutschland die Implementierungsforschung zu einem eigenen Forschungsfeld entwickelt und verbreitet (Wensing und Grol 2019).

Für diese Zielgruppe der Kinder und Jugendlichen psychisch erkrankter Eltern wurde in CHIMPS-NET als bundesweitem Verbund mit 20 klinischen Standorten (immer in Vernetzung zwischen Kliniken für Psychiatrie und für Kinder- und Jugendpsychiatrie und regional unter Beteiligung der Jugendhilfe) erstmals in Deutschland in 


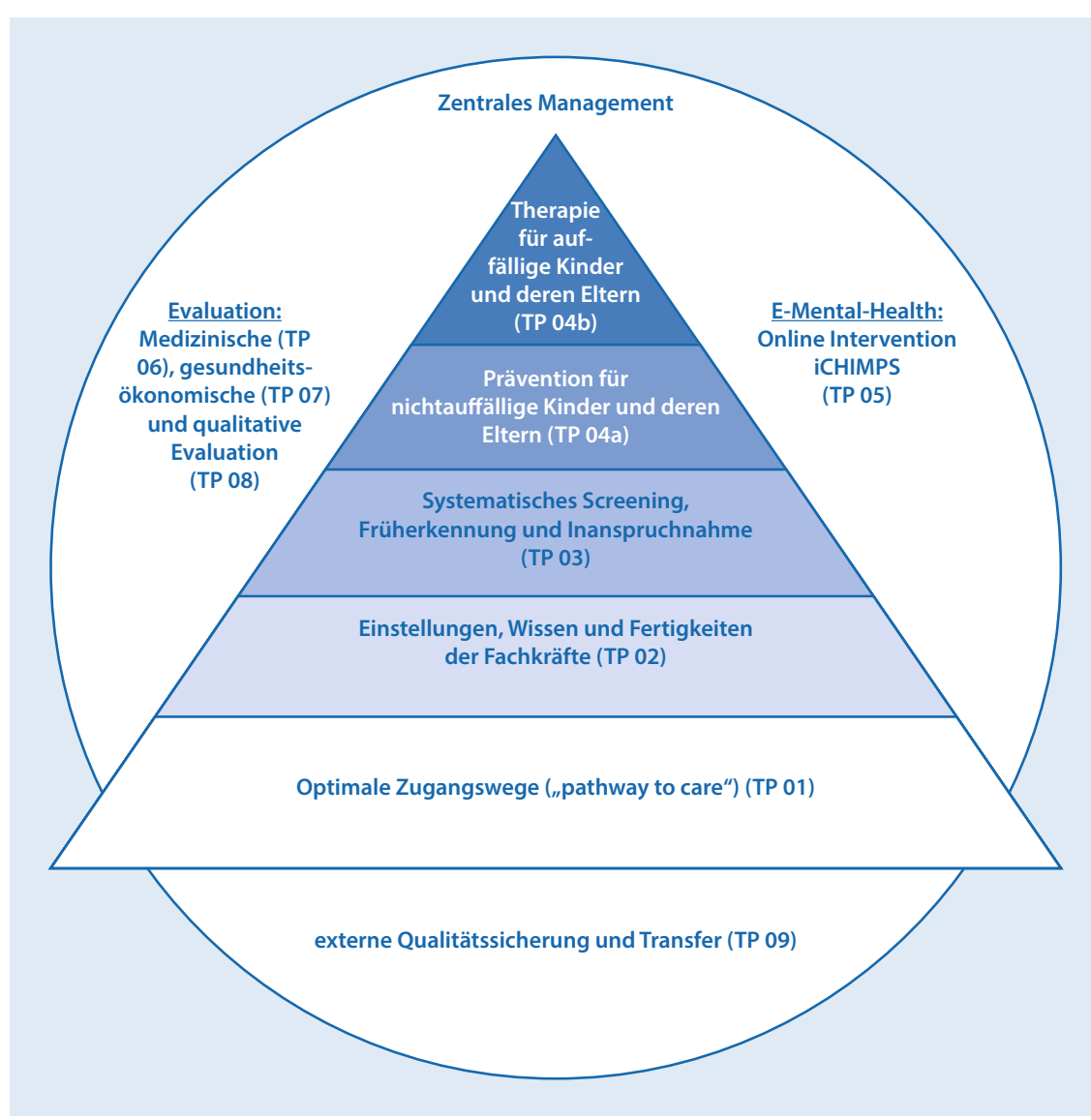

Abb. 1 ॥ Implementierungsmodell CHIMPS-NET zur sektorenübergreifenden, indikationsgeleiteten und schweregradgestuften Versorgung von Kindern und Jugendlichen im Alter von 0 bis 21 Jahren und ihre psychisch kranken und suchtkranken Eltern. TP Teilprojekt

dieser Größenordnung ein systematisches Implementierungsmodell entwickelt und in RCT-Implementierungsstudien geprüft.

Das Implementierungsmodell CHIMPSNET basiert auf wegweisenden Arbeiten australischer Wissenschaftler, die als Pioniere der Implementierungsforschung für diese Zielgruppe gelten können: Darryl Maybery und Andrea Reupert (z. B. Maybery und Reupert 2009). Im Implementierungsmodell CHIMPS-NET werden die Erkenntnisse dieser australischen Arbeiten, die auch über Skandinavien (z. B. Norwegen) nach Europa gelangten (z. B. Lauritzen und Reedtz 2015) in 3 systematisch aufeinander abgestimmte Implementierungsinterventionen übersetzt und als 3 Teilprojekte (TP) im Verbund realisiert.

\section{Implementierungsmodell CHIMPS- NET}

(•Abb. 1)
Die 3 Implementierungsinterventionen werden als 3 Teilprojekte (TP 01-03) durchgeführt, und zwar: T01:optimale Zugangswege (ein Teilprojekt unter Projektleitung von Silke Pawils, Hamburg); TP 02: Wissen und Fertigkeiten der Fachkräfte (unter Projektleitung von Svenja Taubner, Heidelberg) und TP 03: systematisches Screening (unter Projektleitung von Sibylle Maria Winter, Berlin). Eine übergeordnete Implementierungsstudie ci-Chimps evaluiert den Erfolg dieser systematischen Implementierungsinterventionen und -projekte in einem multizentrischen RCT-Design, das im Studienprotokoll beschrieben wird (Laser et al. subm.). Zwei dieser Implementierungsinterventionen aus CHIMPSNET werden im vorliegenden Heft ausführlicher beschrieben (vgl. die Beiträge von Tauber et al. und von Winter et al.).

Diesen Implementierungsprojekten folgen im Modell die präventiven und therapeutischen Interventionen selbst (TP 04a, b). Zudem wird die CHIMPS-
Intervention (unter Leitung von Harald Baumeister) als Online-Intervention für Jugendliche iCHIMPS weiterentwickelt (TP 05).

Alle 4 Interventionen werden begleitend medizinisch-biometrisch, gesundheitsökonomisch und qualitativ (TP 06-08) unter Leitung von Antonia Zapf, Jan Zeidler \& Johann-Matthias Graf von der Schulenburg, Reinhold Kilian sowie Martin Heinze \& Sebastian von Peter evaluiert. Eine externe klinische Qualitätssicherung (unter Leitung von Gerald Willms, aQua Institut, Göttingen, TP09) begleitet den Transfer der Interventionen unter klinischer Versorgungsperspektive und entwickelt ein Qualitätsmodell für alle Interventionen.

Neue Versorgungsformen als Teil der Gesundheitsversorgung Schnittstelle zwischen Wissenschaft und Praxis

Der CHIMPS-NET-Verbund will in seiner Ausrichtung verschiedene Ziele erreichen. Es soll zum einen eine hohe Qualität der wissenschaftlichen Untersuchung gewährleistet werden, die sich durch eine methodisch hochwertige und umfassende Evaluation auszeichnet. So sollen beispielsweise neben den biometrischen Wirksamkeitsbelegen auch gesundheitsökonomische Evaluationen vorgelegt werden, konkret die kurz- und langfristigen Kosten sowie die Verbesserung der Lebensqualität, die durch die Interventionen erreicht werden. Hierzu wird mit 4 Partnern aus den Bereichen Biometrie, Gesundheitsökonomie, Qualitätssicherung und qualitative Evaluation zusammengearbeitet. Für Details zur Methodik der multizentrischen Evaluationsstudien wird nochmals auf das Studienprotokoll verwiesen (Wiegand-Grefe et al. subm.). Für ein korrektes und datenschutzsicheres Datenmanagement und -monitoring wird ein externes "clinical trial center" (CTC North) beauftragt. Um das Angebot bekannt zu machen, und um eine möglichst breite Zielgruppe zu erreichen, wird mit Partnern und Partnerinnen der Öffentlichkeitsarbeit (Homepage: Jörg Dirmaier) sowie der Vernetzung mit der Jugendhilfe (AFET, Koralia Sekler) und Gemeindepsychiatrie (Birgit Görres, Nils Greve) eng zusammengearbeitet. Um diese familie- 


\section{Infobox 1}

Die Interventionen in CHIMPS-NET richten sich passgenau nach dem Bedarf der Familie und bieten je nach gesundheitlicher Ausgangslage eine Prävention, eine Gruppenintervention oder eine Therapie. Alle Konzepte und Interventionen sind diagnoseübergreifend und in einem weiten Altersbereich ab der Geburt bis ins junge Erwachsenenalter einsetzbar.

norientierten Versorgungsformen in die Versorgung der gesetzlichen Krankenkassen aufzunehmen, wurde der CHIMPSNET-Verbund, wie im Innovationsfonds üblich, so konzipiert, dass von Projektbeginn an gesetzliche Krankenkassen als gleichberechtigte Verbundpartner gewonnen wurden. Mit allen teilnehmenden Krankenkassen (Techniker Krankenkasse, BARMER, DAK, Mobil Krankenkasse, Kaufmännische Krankenkasse, IKK Classic, AOK Baden-Württemberg und AOK Hessen als Konsortialpartner, weitere Krankenkassen sind dem Selektivvertrag und dem Verbund als Kooperationspartner beigetreten) wurden zu Projektbeginn Selektivverträge nach § 140a SGB V abgeschlossen. Bei erfolgreicher Evaluation des Projekts sollen diese mit den Krankenkassen für die Projektlaufzeit abgeschlossenen Selektivverträge verlängert werden. Damit wären die CHIMPS-Interventionen als neue Versorgungsformen Teil der selektivvertraglichen Versorgung der teilnehmenden gesetzlichen Krankenkassen. Dieses Vorgehen zeichnet den Verbund CHIMPSNET aus: zusätzlich zur fundierten wissenschaftlichen Untersuchung wird von Beginn an die Versorgungsnähe berücksichtigt. Das heißt, eine zeitnahe Überführung der wissenschaftlichen Ergebnisse in die Regelversorgung ist explizit vorgesehen. Damit realisiert der CHIMPS-NETVerbund eine enge Verknüpfung zwischen Wissenschaft und Praxis - hier konkret eine verbesserte Versorgungssituation der Kinder und Jugendlichen mit psychisch erkrankten Eltern.

\section{Klinischer Ansatz}

Im Folgenden wird am Beispiel des CHIMPS-Ansatzes beschrieben, wie sich neue, familienorientierte Versorgungsformen in das Gesundheitssystem integrieren lassen können. Es geht darum, ein versorgungsnahes und breit einsetzbares Angebot zu schaffen. Mit dem CHIMPSAnsatz wird beispielhaft ein Präventionsund Therapieprogramm für Kinder und Jugendliche mit psychisch erkrankten Eltern und ihrer Familien vorgestellt.

Der CHIMPS-Ansatz basiert konzeptuell auf 3 Säulen: 1. auf einem Theoriemodell (Wiegand-Grefe et al. 2011a), 2. auf Bedarfsanalysen betroffener Familien (Wiegand-Grefe 2010) und 3. auf der psychoanalytischen Familientherapie. Das Theoriemodell definiert gemäß einem Modell von Mattejat et al. (2000) die beiden Säulen Krankheitsbewältigung und Familienbeziehungen, aus denen therapeutische Ansatzpunkte der Interventionen abgeleitet werden. In Bedarfsanalysen wurde ermittelt, dass sich von einer psychischen Erkrankung betroffene Eltern den Einbezug der gesamten Familie in die Behandlung wünschen. Um das Angebot möglichst vielen Kindern und Jugendlichen mit psychisch erkrankten Eltern und ihren Familien zugänglich zu machen, wurde es diagnoseübergreifend entwickelt. Darüber hinaus ist es für einen breiten Altersbereich von Kindern und Jugendlichen ab der Geburt bis ins junge Erwachsenenalter einsetzbar. Das Konzept ist also unabhängig von der elterlichen Diagnose ab der Geburt eines Kindes bis zum jungen Erwachsenenalter anwendbar, sofern die Bedingungen für eine ambulante Behandlung gegeben sind. Bei Bedarf kann das Konzept auch aufsuchend durchgeführt werden. Es handelt sich um ein niederfrequentes Konzept, dass sich über ein halbes Jahr erstreckt. Es kann verschiedene Behandlungen von einzelnen Familienmitgliedern unkompliziert begleiten oder auch diesen vorausgehen. Für erkrankte Eltern im stationärem Behandlungskontext wird mit CHIMPS-NET eine ambulante Nachsorge für die ganze Familie realisiert.

Das Grundkonzept des CHIMPS-Ansatzes ist in einem Manual beschrieben (Wiegand-Grefe et al. 2011a), daher wird an dieser Stelle nur zusammenfassend auf die Inhalte verwiesen. Der CHIMPS-Ansatz hat sich im Setting der 6 bis 8 Sitzungen auch von den Arbeiten von Beardslee (2009) zu depressiven Eltern anregen lassen, hat jedoch andere Gesprächsinhalte und therapeutische Interventionen. Der
Ansatz erweitert damit Beardslees Ansatz um eine psychodynamische Perspektive, um psychodynamische Interventionstechniken, psychodynamische Ziele sowie um den Gegenstand und die Zielgruppe (er ist störungsübergreifend für alle elterlichen Diagnosegruppe und eine breite Altersgruppe der Kinder konzipiert). Die 3 übergeordneten Ziele der CHIMPS-Intervention sind:

- Ein Verständnis der Erkrankung sowie der Paar- und Familiendynamik aus einer psychodynamischen, mehrgenerationalen Perspektive, um die transgenerationale Dynamiken für Familien und Therapeuten transparenter und verstehbarer zu machen.

- Eine angemessene Krankheitsbewältigung, die bei Bedarf beispielsweise eine altersgerechte Information und Aufklärung aller Familienmitglieder über die elterliche Erkrankung beinhalten kann. Hier kann auf bewährte Psychoedukationselemente, wie z. B. passende Kinderbücher, zurückgegriffen werden.

- Eine Verbesserung der innerfamiliären und außerfamiliären Beziehungen und des sozialen Netzwerkes der Familie. So kann z. B. gemeinsam überlegt werden, welche Bezugspersonen ergänzend für das Kind da sein können, um eine Entlastung des erkrankten Elternteils bei gleichzeitiger Unterstützung des Kindes herbeizuführen.

Der CHIMPS-Ansatz ist seit nunmehr 10 Jahren gut evaluiert (Wiegand-Grefe et al. 2021). Er verbessert nachweislich die psychische Gesundheit und die Lebensqualität der Kinder, die Krankheitsbewältigung und die innerfamiliären Beziehungen (z.B. Wiegand-Grefe et al. 2012, 2013).

Im Folgenden wird am Beispiel des CHIMPS-Ansatzes beschrieben, wie sich neue, familienorientierte Versorgungsformen in das Gesundheitssystem integrieren lassen können. Mit den neuen Versorgungsformen auf der Grundlage des CHIMPS-Ansatzes werden mehrere Präventions- und Therapieprogramme für Kinder und Jugendliche psychisch erkrankter Eltern und ihrer Familien vorgestellt und in CHIMPS-NET realisiert. 
Weiterentwicklung des Ansatzes in passgenaue Versorgungsformen

Die CHIMPS-NET-Interventionen sollen möglichst passgenaue Angebote für unterschiedliche Bedarfe der Familien anbieten und damit den sehr unterschiedlichen Ausgangslagen Rechnung tragen (- Infobox 1). Sie sind versorgungsnah und bieten deshalb Unterstützungsmaßnahmen in unterschiedlicher Intensität an - ganz nach Bedarf und klinischer Indikation, also je nach Indikation und gesundheitlicher Ausgangslage der Familien:

- eine kurze Prävention, CHIMPS-P, für funktionale Familien mit belasteten, aber psychisch gesunden Kindern,

- die CHIMPS-MFT für belastete Familien mit Kindern, auch wenn diese bereits eigene Auffälligkeiten zeigen und die Familien vom Gruppenformat profitieren können,

- und die therapeutische Intervention, CHIMPS-T, für weniger funktionale Familien, für die ein Gruppenformat nicht geeignet ist.

In den folgenden Abschnitten werden die 4 Interventionsformen CHIMPS-T, CHIMPSMFT, CHIMPS-P und iCHIMPS skizziert. Für weitergehende Informationen wird wieder auf die Studienprotokolle verwiesen (Wiegand-Grefe et al. subm.; Dülsen et al. subm.). Die CHIMPS-NET-Interventionen werden an 20 Standorten (zumeist in Kooperation zwischen einer Klinik für Erwachsenenpsychiatrie, einer Klinik für Kinder- und Jugendpsychiatrie und der Jugendhilfe) in Deutschland in 15 Bundesländern angeboten.

CHIMPS-T - therapeutisches Angebot für Familien mit hohem Unterstützungsbedarf

An dieser Stelle erfolgt lediglich ein Überblick, um ein grobes Verständnis für die Intervention zu schaffen: An ein Erstgespräch mit der Familie schließen sich 2 bis 3 Eltern- bzw. Paargespräche an und danach Einzelgespräche für jedes Kind der Familie. Den Abschluss bilden 3 Gespräche mit der gesamten Familie. Das Angebot CHIMPS-T umfasst also 3 Abschnitte mit Eltern-, Kind- und Familiengesprächen, die sich über ca. 8 Sitzungen erstrecken. Die
Sitzungen werden niederfrequent in 2-bis 3-wöchigen Abständen angeboten. Die familientherapeutische Intervention findet über ein halbes Jahr hinweg statt. Zur ausführlichen Beschreibung wird auf das oben genannte Manual von Wiegand-Grefe et al. (2011a) verwiesen. Inhaltlich werden stets die Krankheitsbewältigung und die Familienbeziehungen adressiert sowie Verbesserungsmöglichkeiten erarbeitet. Ziel der Familiengespräche ist es, die unterschiedlichen Sichtweisen, neu gewonnene Erkenntnisse und Wünsche der Familienmitglieder zusammenzutragen. Wird die elterliche psychische Erkrankung besprochen, kann die Therapeutin bei der Aufklärung des Kindes unterstützen. Den Abschluss bilden weitergehende Empfehlungen an die Familie, wie z. B. die Empfehlung einer psychotherapeutischen Behandlung des Kindes oder des Partners bzw. der Partnerin. Aktuell wird der Ansatz für Eltern mit Persönlichkeitsstörungen und anderen schweren Störungen unter dem Einfluss der übertragungsfokussierten Psychotherapie („transference-focused psychotherapy, TFP"; Yeomans et al. 2017) weiterentwickelt.

CHIMPS-MFT - multifamilientherapeutisches Gruppenangebot für belastete Familien

Die Intervention CHIMPS-MFT erstreckt sich ebenfalls über 8 Sitzungen. Im multifamilientherapeutischen Setting werden die Themen „Krankheitsbewältigung" und "Familienbeziehungen“ bearbeitet, zusätzlich werden die Ressourcen der Familienmitglieder betrachtet. Grundlage dieser multifamilientherapeutischen Arbeit ist die mehrgenerationale Arbeit mit dem Genogramm. Der Ansatz ist in einem Manual beschrieben (Wiegand-Grefe et al. 2020).

CHIMPS-P - präventives Angebot für gut funktionierende Familien

Die kurze Prävention CHIMPS-P aus 3 Sitzungen orientiert sich inhaltlich an den 3 Familiengesprächen des Chimps-Ansatzes (Manual: Wiegand-Grefe et al. 2011a). Aufgrund der hohen Funktionalität der Familie wird auf die Eltern- und Einzelgespräche mit den Kindern verzichtet. Sollten sich jedoch Hinweise auf einen erhöhten Unterstützungsbedarf geben, kann da- rauf selbstverständlich eingegangen werden. Die Prävention CHIMPS-P mit 3 Familiengesprächen setzt keine Approbation als Qualifikation voraus, sondern kann beispielsweise auch von SozialpädagogInnen mit familientherapeutischer Weiterbildung durchgeführt werden.

\section{iCHIMPS}

Ferner wird in CHIMPS-NET unter der Projektleitung von Harald Baumeister, Ulm, eine auf dem CHIMPS-Modell basierende Online-Intervention, iCHIMPS, für Jugendliche mit psychisch erkrankten Eltern entwickelt (https://esano.klips-ulm.de/ de/trainings/fur-jugendliche/ichimps/).

Mit iCHIMPS wird eine Intervention für belastete Jugendliche bereitgestellt, deren Eltern nicht an familienorientierten Interventionen interessiert sind.

\section{Fazit}

- Die 4 Interventionsformen nach dem "Children-of-mentally-ill-parents"

(CHIMPS)-Ansatz stellen ein bedarfsorientiertes Vorgehen dar, das diagnoseübergreifend und in einem breiten Altersbereich einsetzbar ist.

- Alle 4 Interventionen werden in jeweils eigenen "randomized controlled trials" (RCT) evaluiert, das Design dieser RCT wird in den Studienprotokollen publiziert.

- Im Fall einer positiven Evaluation würde die Versorgung über die Selektivverträge in die Routineversorgung gelangen. Damit würde ein ausdrücklich familienorientierter Ansatz in die regelhafte Versorgung psychisch Erkrankter aufgenommen werden. Das wäre ein wichtiger Schritt von einem individuumszentrierten Vorgehen zu einer stärker familienorientierten Sichtweise.

\section{Korrespondenzadresse}

Prof. Dr. rer. nat Dipl.-Psych.

Silke Wiegand-Grefe

Klinik für Kinder- und Jugendpsychiatrie,

-psychotherapie und -psychosomatik, Zentrum für Psychosoziale Medizin, Universitätsklinikum Hamburg Eppendorf (UKE)

Martinistr. 52, W35, 20246 Hamburg,

Deutschland

swiegand-grefe@uke.de

Danksagung. Ein großes Dankeschön allen beteiligten Konsortialpartnern. Aus den Kliniken sind an der Implementierung der Interventionen beteiligt: Jürgen Gallinat, Anne Karow (Hamburg), Reinhold 
Kilian \& Thomas Becker (Ulm-Günzburg), Stephan Bender \& Frank Jessen (Köln), Michael Siniatchkin \& Martin Driessen (Bielefeld), Michael Kölch (Rostock), Sibylle Maria Winter, Fritz Bermpohl \& Andreas Heinz (Berlin), Henning Flechtner \& Thomas Frodl (Magdeburg), Ekkard Englert \& Ralph Schlößer (Erfurt), Katja Becker \& Tilo Kircher (Marburg), Andreas Reif (Frankfurt), Christian Fleischhaker \& Katarina Domschke (Freiburg), Michele Noterdaeme, Max Schmauß \& Markus Jäger (Augsburg/Kempten), Gerd Schulte-Körne \& Belinda Weber (München), Tobis Renner \& Andreas Fallgatter (Tübingen), Eva Möhler \& Ulrich Seidl (Homburg), Tina In-Albon, Michael Brünger \& Sylvia Claus (Landau), Martin Heinze \& Roland Burkhard (Rüdersdorf/Frankfurt/Oder), Klaus-Thomas Kronmüller, Fabian Klein \& Martin Holtmann (Gütersloh-Hamm), Christiane RummelKluge (Leipzig), Simone Born (Karlsruhe), Christian Haase \& Andreas Broocks (Schwerin). Als Evaluatoren sind beteiligt: Antonia Zapf (Hamburg), Jan Zeidler \& Johann-Matthias Graf von der Schulenburg (Hannover), Reinhold-Kilian (Ulm-Günzburg), Martin Heinze \& Sebastian von Peter (Brandenburg), Gerald Willms (Göttingen). Die Implementierungsprojekte realisieren: Svenja Taubner, Sibylle Maria Winter, Silke Pawils. Als Vernetzungspartner zur Jugendhilfe, Gemeindepsychiatrie und Öffentlichkeit sind tätig: Birgit Görres, Jutta Decarli, Koralia Sekler, Jörg Dirmeier. Die Online Intervention iCHIMPS realisiert: Harald Baumeister. Die face to face Interventionen CHIMPS-P, CHIMPS-T und CHIMPS-MFT supervidiert die Studienzentrale. Zum Team der Studienzentrale gehören aktuell: Joana Taczkowski, Anna Leidger, Ann-Katrin Meyer, Paul Lang, Anna Modaressi.

\section{Einhaltung ethischer Richtlinien}

Interessenkonflikt. S. Wiegand-Grefe gibt an, dass kein Interessenkonflikt besteht.

Für diesen Beitrag wurden von der Autorin keine Studien an Menschen oder Tieren durchgeführt. Für die aufgeführten Studien gelten die jeweils dort angegebenen ethischen Richtlinien.

Open Access. Dieser Artikel wird unter der Creative Commons Namensnennung 4.0 International Lizenz veröffentlicht, welche die Nutzung, Vervielfältigung, Bearbeitung, Verbreitung und Wiedergabe in jeglichem Medium und Format erlaubt, sofern Sie den/die ursprünglichen Autor(en) und die Quelle ordnungsgemäß nennen, einen Link zur Creative Commons Lizenz beifügen und angeben, ob Änderungen vorgenommen wurden.

Die in diesem Artikel enthaltenen Bilder und sonstiges Drittmaterial unterliegen ebenfalls der genannten Creative Commons Lizenz, sofern sich aus der Abbildungslegende nichts anderes ergibt. Sofern das betreffende Material nicht unter der genannten Creative Commons Lizenz steht und die betreffende Handlung nicht nach gesetzlichen Vorschriften erlaubt ist, ist für die oben aufgeführten Weiterverwendungen des Materials die Einwilligung des jeweiligen Rechteinhabers einzuholen.

Weitere Details zur Lizenz entnehmen Sie bitte der Lizenzinformation auf http://creativecommons.org/ licenses/by/4.0/deed.de.

\section{Literatur}

Baumeister H (2014) Implementationsforschung in der Klinischen Psychologie, Rehabilitationspsychologie und Psychotherapie. Psychol Rundsch 65:150-158. https://doi.org/10.1026/ 0033-3042/a000217 (Online veröffentlicht: July $08,2014)$

Beardslee WR (2009) Hoffnung, Sinn und Kontinuität: Ein Programm für Familien depressiv erkrankter Eltern. dgvt, Tübingen. ISBN 978-3-87159-619-3

Dülsen P, Barck K, Daubmann A, Höller A, Kilian R, Wiegand-Grefe S, Baumeister H (subm.) Clinical-and cost effectiveness of a guided internetbased intervention for children (12 to 18 years) of parents with mental disorders (iCHIMPS): Study protocol of a multicentered cluster-randomized controlled trial. Frontiers in Digital Health

Grube M, Dorn A (2007) Parenthood and mental illness. Psychiat Prax 34:66-71

Hatzinikolaou K, Murray L (2010) Infant sensitivity to negative maternal emotional shifts: effects on infant sex, maternal postnatal depression, and interactive style. Infant Ment Health J 31:591-610

Jordan W, Bielau H, Cohrs S, Hauth I, Hornstein C, Marx A, Reck C, von Einsiedel R (2012) Aktuelle Versorgungs- und Finanzierungslage von Mutter-Kind-Einheiten für schwangerschaftsassoziierte psychische Störungen in Deutschland. Psychiatr Prax 39(05):205-210. https://doi.org/ 10.1055/s-0032-1304918

Laser C, Skogøy BE, Reupert A, Taubner S, Winter S, Pawils S, Daubmann A, Zapf A, Maybery D, Wiegand-Grefe S (subm.) Clinical implementation of the family-oriented intervention for children of mentally ill parents (ci-chimps): study protocol for a randomized controlled implementation multicentre trial

Lauritzen C, Reedtz C (2015) Knowledge transfer in the field of parental mental illness: objectives, effective strategies, indicators of success, and sustainability. Int J Ment Health Syst. https:// doi.org/10.1186/1752-4458-9-6 (http://www. ijmhs.com/content/9/1/6)

Mäntymaa M, Puura K, Luoma I et al (2004) Early mother-infant interaction, parental mental health and symptoms of behavioural and emotional problems in toddlers. Infant Behav Dev 2004(27):134-149

Mattejat F, Remschmidt H (2008) Kinder psychisch kranker Eltern. Dtsch Arztebl 2008(23):413-418

Mattejat M, Wüthrich C, Remschmidt H (2000) Kinder psychisch kranker Eltern. Forschungsperspektiven am Beispiel von Kindern depressiver Eltern. Nervenarzt 71:164-172. https://doi.org/ $10.1007 / \mathrm{s} 001150050025$

Maybery DJ, Reupert AE (2009) Parental mental illness: a review of barriers and issues for working with families and children. J Psychiatr Ment Health Nurs 16(9):784-791

Müller JM, Averbeck-Holocher M, Romer G, Fürniss T, Achtergarde S, Postert C (2015) Psychiatric treatment outcomes of preschool children in a family day hospital. Child Psychiatry Hum Dev 46:257-269

Petermann F (2014) Implementationsforschung: Grundbegriffe und Konzepte. Psychol Rundsch 65(3):122-128. https://doi.org/10.1026/00333042/a000214

Petermann U, Petermann F (2006) Erziehungskompetenz. Kindh Entwickl 15:1-8

Radicke A, Barkmann C, Adema B, Daubmann A WegscheiderK, Wiegand-Grefe S(2021)Children with mentally ill parents: predictors of healthrelated quality of life and determinants of childparent agreement. IJERPH 18(2):379-2021. https://doi.org/10.3390/ijerph18020379

Ravens-Sieberer U, Wille N, Bettge S, Erhart M (2007) PsychischeGesundheitvon Kindern und Jugendlichen in Deutschland. Ergebnisse aus der BELLAStudie im Kinder- und Jugendgesundheitssurvey (KiGGS). Bundesgesundheitsblatt Gesundheitsforschung Gesundheitsschutz 50:871-878. https://doi.org/10.1007/s00103-007-0250-6

Sell M, Daubmann A, Zapf H, Adema B, Busmann M, Stiawa M, Winter SM, Lambert M, Wegscheider K, Wiegand-Grefe S (2021) Family functioning in families affected by parental mental illness: parent, child, and clinician ratings. IJERPH 18:7985. https://doi.org/10.3390/ijerph18157985

Smeekens S, Riksen-Walraven JM, van Bakel HJA (2007) Multiple determinants of externalizing behavior in 5-years old: a longitudinal model. J Abnorm Child Psychol 2007(35):347-361

Steffen A, Akmatov MK, Holstiege J, Bätzing J (2018) Diagnoseprävalenz psychischer Störungen bei Kindern und Jugendlichen in Deutschland: eine Analyse bundesweiter vertragsärztlicher Abrechnungsdaten der Jahre 2009 bis 2017. Zentralinstitut für die kassenärztliche Versorgung in Deutschland, Berlin. https://doi.org/10.20364/ VA-18.07

Tronick E, Reck C (2009) Infants of depressed mothers. Harv Revue Psychiatry 17:147-156

Wensing M, Grol R (2019) Knowledge translation in health: how implementation science could contribute more. BMC Med 17:88. https://doi. org/10.1186/s12916-019-1322

Wiegand-Grefe S (2010) Die Belastungen von Kindern psychisch kranker Eltern. Neurol Psychiater 11:42-47

Wiegand-Grefe S, Taczkowski J (2021) Familie mitdenken - Plädoyer für eine Reform des Versorgungssystems hin zur Familienmedizin und Familienpsychologie. PiD 22:19-24

Wiegand-Grefe S, Geers P, Plass A et al (2009) Children of mentally ill parents: associations between subjective parental impairment and psychological problems of the children. Kindh Entwickl 18:111-121. https://doi.org/10.1026/ 0942-5403.18.2.111

Wiegand-Grefe S, Halverscheid S, Plass A (2011a) Kinder und ihre psychisch kranken Eltern. Familienorientierte Prävention - der CHIMPsBeratungsansatz. Hogrefe, Göttingen

Wiegand-Grefe S, Geers P, Petermann F, Plass A (2011b) Kinder psychisch kranker Eltern: Merkmale elterlicher psychiatrischer Erkrankung und Gesundheit der Kinder aus Elternsicht. Fortschritte Neurol Psychiatr 79:32-40. https://doi.org/10. 1055/s-0029-1245623

Wiegand-Grefe S, Werkmeister S, Bullinger M, Plass A, Petermann F (2012) Health-related quality of life and social support of children with mentally ill parents: effects of a manualized family intervention. Kindh Entwickl 21:64-73. https:// doi.org/10.1026/0942-5403/a000071

Wiegand-Grefe S, Cronemeyer B, Plass A, SchulteMarkwort M, Petermann F (2013) Comparison of mental abnormalities in children of mentally ill parents from different points of view: effects of a manualized family intervention. Kindh Entwickl 22(1):31-40. https://doi.org/10.1026/ 0942-5403/a000096

Wiegand-Grefe S, Sell M, Filter B, Plass-Christl A (2019) Family functioning and psychological health of children with mentally ill parents. 
IJERPH 16(7):1278. https://doi.org/10.3390/ ijerph16071278

Wiegand-Grefe S, Sell M, Plass-Christl A (2020) Die Gruppenangebote nach dem CHIMPS-Modell (projektinternes Material, unveröffentlichtes Manuskript)

Wiegand-Grefe S, Filter B, Busmann M, Kilian R, Kronmüller K-T, Lambert M, Norra C, von Klitzing K, Albermann K, Winter SM, Daubmann A, Wegscheider K, Plaß-Christl A (2021) Evaluation of a family-based intervention program for children of mentally ill parents: study protocol for a randomized controlled multicenter trial. Front Psychiatry 11:561790. https://doi.org/10.3389/ fpsyt.2020.561790

Wiegand-Grefe S, Taczkowski J, Leidger A, Modaressi A, Meyer AK, Adema B, Hot A, Daubmann A, Zapf A, Dirmaier J, Pawils S, Gallinat J, Karow A, Kilian R, Becker T, Zeidler J, von der Schulenburg M, Taubner S, Willms G, Goerres B, Decarli J, Sekler J, Bender $S$, Jessen $F$, Siniatchkin $M$, Drießen $M$, Kölch $M$, Winter $S$, Bermpohl $F$, Heinz A, Flechtner $H$, Frodl $T$, Englert $E$, Schlößer R, Becker K, Kircher T, Reif A, Leibing E, Reich G, Kis B, Fleischhaker C, Domschke K, Noterdaeme M, Schmauß M, Jäger M, SchulteKörne $G$, Weber B, Renner T, Fallgatter A, Möhler E, Seidl U, In-Albon T, Brünger M, Claus S, Heinze $M$, von Peter $S$, Burkhard R, Klein $F$, Kronmüller KT, Holtmann M, Rummel-Kluge C, Haase C, Brooks A, Born S, Baumeister H (subm.) Evaluation of four tailrored, need-adapted (face to face and online) interventions for children and adolescents of mentallyill parens-research network (CHIMPS-NET): study protocol for four randomized controlled multicenter trials

Yeomans FE, Clarkin JF, Kernberg OF (2017) ÜbertragungsfokussiertePsychotherapiefürBorderlinePatienten. Das TFP-Praxismanual. Schattauer, Stuttgart

\section{Support for children and adolescents with mentally ill parents in the "Children of mentally ill parents—network". Nationwide research, care and implementation model}

Children of mentally ill parents are known to be a risk group for developing their own psychopathology. Nonetheless, many support offers have a limited project character and no family-oriented standard of care has yet been established for children and adolescents of mentally ill parents in Germany. Using the association "Children of mentally ill parents-network" (CHIMP-NET) as an example, the aim is to show how forms of interventions can be implemented in care with the possibilities of the Federal Joint Committee (GB-A) innovation fund. The systematic implementation model of the network, based on work from Australia and Norway, is presented. After implementation and successful evaluation, it is planned to implement the needs-based, family-oriented forms of care of the network into standard care.

\section{Keywords}

Family-oriented standard care · Prevention · Psychotherapeutic interventions · Implementation research - Evaluation 